\title{
General Solutions of Nonlinear Equations in the Geometric Theory of the Relativistic String
}

\author{
B. M. Barbashov, V. V. Nesterenko, and A. M. Chervyakov \\ Laboratory of Theoretical Physics, Joint Institute for Nuclear Research, SU-141980 Dubna, USSR
}

\begin{abstract}
General solutions for the system of nonlinear equations in the second order partial derivatives with two independent variables are obtained. They determine the basic differential forms of the two-dimensional minimal surface embedded into $n$-dimensional pseudo-Euclidean space.
\end{abstract}

\section{Introduction}

In recent years a good deal of attention has been paid to the derivation of exact solutions for the nonlinear equations in partial derivatives. In elementary particle theory this interest stems from attempts to go outside the limits of perturbation theory in the quantum field approach (solitons, instantons, strings, etc. $[1-4]$ ). For the two-dimensional field models the inverse scattering method turned out to be effective for these investigations [5].

A series of papers [6-11] gives geometric interpretation of the nonlinear equations solved by the inverse scattering method. It has been shown that these equations are tightly related with the intrinsic geometry of surfaces in the Euclidean, pseudo-Euclidean and affine spaces (pseudo-spheres, minimal surfaces, surfaces of a constant mean curvature, etc.). Moreover, the linear equations describing the change of the moving basis during the motion of its origin along the surface can be considered as the Lax operators for the relevant nonlinear equation.

In this paper we shall deal with the nonlinear equations describing in differential geometry the minimal surfaces in the pseudo-Euclidean space. The geometric nature of these equations allows one to obtain explicitly their general solutions.

We shall consider two different parametrizations of the minimal surfaces, and as a consequence, we shall obtain two series of the systems of nonlinear equations. The general solutions of these equations will be found exactly. The first series starts with the nonlinear Liouville equation (3.8), then we have the system of two equations (3.16), the new system of three nonlinear equations (3.28), and so on. The other parametrization of the minimal surfaces (the so-called $t=\tau$ gauge) leads to the different series of systems beginning with the D'Alambert equation (4.8). 
The next system here is a modified version of the Lund-Regge equations (4.13). The general solutions of equations of the first series are given by Eqs. (3.9), (3.17), (3.19) and (3.29) and of the second series by Eqs. (4.12), (4.16) and (4.17).

The theory of minimal surfaces has a long history originating from the papers of Lagrange (1760) and the famous Plateau problem. In recent years the minimal surfaces have appeared also in the theoretical apparatus of elementary particle physics. First of all, this is the relativistic string model dealing with a onedimensional relativistic object, whose world surface is a minimal surface in Minkowski space $[2,4]$. The notion of minimal surface is used to formulate the Wilson criterion of quark confinement in gauge models of strong interactions [12].

\section{Relativistic String Model. Equations of Motion for the String Coordinates and Their Solutions}

This model is the one-dimensional object with the action proportional to the area of its world surface in Minkowski space [4]. If $x^{\mu}(\tau, \sigma)$ are the coordinates of the world surface of the string, then the action of the string is defined by

$$
S=-\gamma \int_{\tau_{1}}^{\tau_{2}} d \tau \int_{\sigma_{1}}^{\sigma_{2}} d \sigma \sqrt{\left(\dot{x} x^{\prime}\right)^{2}-\dot{x}^{2} x^{\prime 2}}=-\gamma \iint d^{2} u \sqrt{-g(u)}
$$

where $\dot{x}^{\mu}=\partial x^{\mu} / \partial \tau, x^{\prime \mu}=\partial x^{\mu} / \partial \sigma, g=\operatorname{det}\left\|g_{i j}\right\|, g_{\imath j}=\frac{\partial x^{\mu}}{\partial u^{i}} \frac{\partial x_{\mu}}{\partial u^{j}}$ is the metric tensor on the string world surface, $u^{1}=\tau$ and $u^{2}=\sigma$. For the proper dimension in (2.1) the constant $\gamma$ with the dimension of inverse squared length is introduced. In Minkowski space we use the metric in which $a^{2}=a^{\mu} a_{\mu}=\left(a^{0}\right)^{2}-\mathbf{a}^{2}$. In the string theory it is assumed that $\dot{x}^{\mu}$ is the time-like vector $\left(g_{11}=\dot{x}^{2}>0\right)$, and $x^{\prime \mu}$ is the space-like vector $\left(g_{22}=x^{\prime 2}<0\right)$ and $g(u)<0$. The principle of least action, as applied to the functional (2.1), requires that the world surface of the string is the minimal surface.

In the isometric coordinate system

$$
g_{11}=\dot{x}^{2}=-g_{22}=-x^{\prime 2}, \quad g_{12}=\dot{x} x^{\prime}=0,
$$

the Euler equations for the action (2.1)

$$
\frac{\delta \sqrt{-g}}{\delta x_{\mu}}=0
$$

are reduced to the D'Alambert equation for the string coordinates

$$
x_{, 11}^{\mu}-x_{, 22}^{\mu}=0
$$

with the boundary conditions

$$
x_{, 2}^{\mu}\left(\tau, \sigma_{1}\right)=x_{, 2}^{\mu}\left(\tau, \sigma_{2}\right)=0 .
$$

The indices with comma in these formulae denote the partial derivatives with respect to the parameters $u^{i}, i=1,2$. In what follows we shall not take into account the boundary conditions (2.5). For simplicity we assume the string to be infinite: $-\infty<u^{i}<+\infty, i=1,2$. 
The equations of motion (2.4) and conditions (2.2) are invariant with respect to the conformal transformations of the parameters $u^{1}, u^{2}: \bar{u}^{ \pm}=f_{ \pm}\left(u^{ \pm}\right)$, $u^{ \pm}=u^{1} \pm u^{2}$. Therefore, without loss of generality, we may consider that the following condition

$$
\left(x_{, 11}^{\mu} \pm x_{.12}^{\mu}\right)^{2}=-q^{2}
$$

is fulfilled, where $q^{2}$ is an arbitrary positive constant.

The general solution of the equations of motion (2.4) and additional conditions (2.2) has the form

$$
x^{\mu}\left(u^{1}, u^{2}\right)=\psi_{+}^{\mu}\left(u^{+}\right)+\psi_{-}^{\mu}\left(u^{-}\right)
$$

$\psi_{+}^{\prime \mu}$ and $\psi_{-}^{\prime \mu}$ being the isotropic vectors

$$
\left(\psi_{ \pm}^{\prime}\right)^{2}=0 .
$$

The prime implies the differentiation with respect to the function argument. Substituting (2.7) into (2.6), we obtain one more condition on $\psi_{ \pm}^{\mu}$

$$
\left(\psi_{ \pm}^{\prime \prime}\right)^{2}=-\frac{q^{2}}{4} .
$$

We treat the two-dimensional minimal surface embedded into $n$-dimensional pseudo-Euclidean space with the metric signature $(+\ldots+\cdots)$. It is obvious that all the formulae mentioned above are trivially generalized to this case: Suffice it to consider that the indices numbering the coordinates of the enveloping space change from 1 to $n$.

The conditions (2.8) and (2.9) can easily be satisfied by expanding the vectors $\psi_{ \pm}^{\prime \mu}$ in a special basis, which is used in the differential geometry to study the isotropic curves $[13,14]$. This basis is formed by two isotropic vectors $e_{1}, e_{2}, e_{i}^{2}=0$, $i=1,2, e_{1} e_{2}=1$ and by $n-2$ space-like vectors $e_{j}, e_{j}^{2}=-1$, where $e_{1} e_{j}=e_{2} e_{j}=0$, $e_{j} e_{k}=0, j \neq k ; j, k=3,4, \ldots, n$. The expansion for $\psi_{ \pm}^{\prime}\left(u^{ \pm}\right)$in this basis can be written as

$$
\begin{aligned}
& \psi_{+}^{\prime}\left(u^{+}\right)=A_{+}\left(u^{+}\right)\left[e_{1}+B_{+}\left(u^{+}\right) e_{2}+\sum_{i=1}^{n-2} f_{i}\left(u^{+}\right) e_{2+\imath}\right], \\
& \psi_{-}^{\prime}\left(u^{-}\right)=A_{-}\left(u^{-}\right)\left[e_{1}+B_{-}\left(u^{-}\right) e_{2}-\sum_{j=1}^{n-2} g_{j}\left(u^{-}\right) e_{2+j}\right] .
\end{aligned}
$$

The fulfillment of condition (2.8) and (2.9) requires that

$$
\begin{gathered}
2 A_{+}=q \cdot\left\{\sum_{i=1}^{n-2}\left[f_{i}^{\prime}\left(u^{+}\right)\right]\right\}^{-1 / 2}, \quad 2 A_{-}=q \cdot\left\{\sum_{j=1}^{n-2}\left[g_{j}^{\prime}\left(u^{-}\right)\right]^{2}\right\}^{-1 / 2}, \\
2 B_{+}=\sum_{i=1}^{n-2} f_{l}^{2}\left(u^{+}\right), \quad 2 B_{-}=\sum_{j=1}^{n-2} g_{J}^{2}\left(u^{-}\right) .
\end{gathered}
$$


Finally we have the following representation for the vectors $\psi_{ \pm}^{\prime}\left(u^{ \pm}\right)$

$$
\begin{aligned}
& \psi_{+}^{\prime}\left(u^{+}\right)=\frac{q}{2 \sqrt{\sum_{i=1}^{n-2}\left[f_{i}^{\prime}\left(u^{+}\right)\right]^{2}}}\left[e_{1}+\frac{1}{2} \sum_{i=1}^{n-2} f_{i}^{2}\left(u^{+}\right) e_{2}+\sum_{i=1}^{n-2} f_{i}\left(u^{+}\right) e_{2+i}\right], \\
& \psi_{-}^{\prime}\left(u^{-}\right)=\frac{q}{2 \sqrt{\sum_{i=1}^{n-2}\left[g_{i}^{\prime}\left(u^{-}\right)\right]^{2}}}\left[e_{1}+\frac{1}{2} \sum_{i=1}^{n-2} g_{i}^{2}\left(u^{-}\right) e_{2}-\sum_{i=1}^{n-2} g_{i}\left(u^{-}\right) e_{2+i}\right] .
\end{aligned}
$$

These formulae give the expression for the metric tensor of the two-dimensional minimal surface in $n$-dimensional pseudo-Euclidean space. It depends on $2(n-2)$ arbitrary functions of one variable [14]:

$$
\begin{aligned}
\left(g_{11}\right)^{-1} & =-\left(g_{22}\right)^{-1}=\left(2 \psi_{+}^{\prime}\left(u^{+}\right) \psi_{-}^{\prime}\left(u^{-}\right)\right)^{-1} \\
& =\frac{4}{q^{2}} \cdot \frac{\left\{\sum_{i=1}^{n-2}\left[f_{i}^{\prime}\left(u^{+}\right)\right]^{2} \sum_{j=1}^{n-2}\left[g_{j}^{\prime}\left(u^{-}\right)\right]^{2}\right\}^{1 / 2}}{\sum_{k=1}^{n-2}\left[f_{k}\left(u^{+}\right)+g_{k}\left(u^{-}\right)\right]^{2}} \equiv \Lambda_{n-2} .
\end{aligned}
$$

\section{Nonlinear Equations in the Theory of Minimal Surfaces and Their General Solutions}

Now we consider the nonlinear equations which are in deep relation with the minimal surfaces. For this aim we shall treat the basic differential forms of the surface.

According to the embedding theorems of the differential geometry [15], the surface can be defined up to its position in the space as a whole by the fundamental surface tensors: the metric tensor $g_{i j}(u)$ and the tensors of second quadratic forms $B_{\alpha \mid \jmath j}(u)$, and the torsion vectors $v_{\alpha \beta \mid i}=-v_{\beta \alpha \mid i}, i, j=1,2, \alpha, \beta=3,4, \ldots, n$, where $n$ is the dimension of the enveloping space. These quantities describe the replacements on the surface of the moving basis, constructed by two tangent vectors $x_{, 1}^{\mu}, x_{, 2}^{\mu}$ and $n-2$ normals $\eta_{\alpha}^{\mu}, \alpha=3,4, \ldots, n$. This is expressed by the following derivative formulae:

$$
\begin{aligned}
& x_{; i j}^{\mu}=-\sum_{\alpha=3}^{n} B_{\alpha \mid i j} \eta_{\alpha}^{\mu}, \\
& \eta_{\alpha, i}^{\mu}=-B_{\alpha \mid i j} g^{j k} x_{, k}^{\mu}-\sum_{\substack{\beta=3 \\
\beta \neq \alpha}}^{n} v_{\beta \alpha \mid i} \eta_{\beta}^{\mu} .
\end{aligned}
$$

The semicolon in these formulae means the covariant differentiation with respect to the metric tensor $g_{i j}$. The functions $g_{i j}(u), B_{\alpha \mid i j}(u)$ and $v_{\alpha \beta \mid i}(u)$ cannot be arbitrary and should satisfy the integrability conditions of the linear equations (3.1) and (3.2) on $x_{, i}^{\mu}, \eta_{\alpha}^{\mu}$, which are defined by the equations of Gauss

$$
R_{i j k \ell}=-\sum_{\alpha=3}^{n}\left(B_{\alpha \mid i k} B_{\alpha \mid \jmath \ell}-B_{\alpha \mid i \ell} B_{\alpha \mid j k}\right),
$$


Peterson-Codazzi

$$
B_{\alpha \mid \jmath j ;}-B_{\alpha \mid i k ; j}=-\sum_{\beta}\left(v_{\beta \alpha \mid k} B_{\beta \mid i j}-v_{\beta \alpha \mid j} B_{\beta \mid i k}\right),
$$

and Ricci

$$
v_{\beta \alpha \mid j ; k}-v_{\beta \alpha \mid k ; j}-\sum_{\gamma}\left(v_{\gamma \beta \mid j} v_{\gamma \alpha \mid k}-v_{\gamma \beta \mid k} v_{\gamma \alpha \mid j}\right)+g^{\ell m}\left(B_{\beta \mid \ell j} B_{\alpha \mid m k}-B_{\beta \mid \ell k} B_{\alpha \mid m J}\right)=0 .
$$

In these equations the Latin indices take values 1,2 and the Greek indices $3,4, \ldots$, . In the left-hand side of the Gauss equation there is the curvature tensor $R_{i j k_{\ell}}$ for the metric $g_{i j}$.

The surface is minimal under the requirement $[15,16]$

$$
\sum_{\imath, j=1}^{2} B_{\alpha \mid i j} g^{i j}=0, \quad \alpha=3,4, \ldots, n .
$$

In the isometric coordinates (2.2) this means that

$$
B_{\alpha \mid 11}=B_{\alpha \mid 22}, \quad \alpha=3,4, \ldots, n .
$$

If the minimal surface is embedded into three-dimensional space-time, then the system of equations (3.3) -(3.5) is reduced to a nonlinear Liouville equation $[9,17,18]$

$$
\varphi_{, 11}-\varphi_{, 22}=2 q^{2} e^{\varphi}
$$

where $g_{11}=-g_{22}=e^{-\varphi}$. Using representation (2.13) with $n=3$ for the metric tensor of the minimal surface, we get the wellknown general solution of the Liouville equation [19]

$$
\mathrm{e}^{\varphi}=\Lambda_{1}=\frac{4}{q^{2}} \cdot \frac{f^{\prime}\left(u^{+}\right) g^{\prime}\left(u^{-}\right)}{\left[f\left(u^{+}\right)+g\left(u^{-}\right)\right]^{2}} .
$$

With increasing dimension of the enveloping space with the minimal surface embedded into it, the number of equations in system (3.3)-(3.5) increases rapidly and the number of functions $g_{i j}, B_{\alpha \mid i j}, v_{\alpha \beta \mid i}$ exceeds the number of equations. However, this system of equations can be considerably simplified by choosing a proper moving basis on the minimal surface. Indeed, the derivation of the Gauss, Peterson-Codazzi and Ricci equations (3.3)-(3.5) will not change if we pass from the basis

$$
x_{, 1}^{\mu}, x_{, 2}^{\mu}, \eta_{3}^{\mu}, \ldots, \eta_{n}^{\mu}
$$

to a new one obtained from $(3.10)$ by the $S O(1,1) \times S O(n-2)$ rotation, which does not mix the tangent space of the surface $\left\{x_{, 1}^{\mu}, x_{, 2}^{\mu}\right\}$ with its normal space $\left\{\eta_{3}^{\mu}, \eta_{4}^{\mu}, \ldots, \eta_{n}^{\mu}\right\}$.

If the dimension of the enveloping space is $n \geqq 4$, then in the space normal to the two-dimensional minimal surface there are two mutual orthogonal space-like vectors $x_{; 11}^{\mu}$ and $x_{; 12}^{\mu}$ (according to (3.1) and (3.7) the vector $x_{; 22}^{\mu}$ coincides with $\left.x_{; 11}^{\mu}\right)$. Indeed, in the metric (2.2) from (2.6) it follows that

$$
\left(x_{, 11}^{\mu} \pm x_{, 12}^{\mu}\right)^{2}=\left(x_{; 11}^{\mu} \pm x_{; 12}^{\mu}\right)^{2}=-\sum_{\alpha=3}^{n}\left(B_{\alpha \mid 11} \pm B_{\alpha \mid 12}\right)^{2}=-q^{2}
$$


i.e. $\left(x_{; 11} x_{; 12}\right)=0$. Therefore, it is natural to direct two normals to the minimal surface along the vectors $x_{; 11}^{\mu}$ and $x_{; 12}^{\mu}$, for instance $\eta_{3}^{\mu}$ along $x_{; 11}^{\mu}$ and $\eta_{4}^{\mu}$ along $x_{; 12}^{\mu}$. Then we immediately obtain

$$
B_{3 \mid 12}=B_{4 \mid 11}=B_{4 \mid 22}=B_{\alpha \mid i j}=0, \quad \alpha=5, \ldots, n ; \quad i, j=1,2 .
$$

To satisfy condition (3.11), we assume

$$
B_{3 \mid 11}=q \cos \frac{\theta}{2}, \quad B_{4 \mid 12}=q \sin \frac{\theta}{2} \text {. }
$$

The Gauss equation (3.3) takes the form

$$
\varphi_{, 11}-\varphi_{, 22}=2 q^{2} e^{\varphi} \cos \theta, \quad e^{-\varphi}=x_{, 1}^{2}=-x_{, 2}^{2},
$$

this equation being valid for any dimension of the enveloping space with the minimal surface embedded into it.

The torsion vector $v_{34 \mid i}, i=1,2$ is determined through the function $\theta\left(u^{1}, u^{2}\right)$ using the Peterson-Codazzi equation (3.4) at $\alpha=3,4$

$$
v_{34 \mid 1}=\frac{\theta_{, 2}}{2}, \quad v_{34 \mid 2}=\frac{\theta_{, 1}}{2} \text {. }
$$

In four-dimensional space-time the system (3.3)-(3.5) is reduced to two nonlinear equations

$$
\begin{aligned}
& \varphi_{, 11}-\varphi_{, 22}=2 q^{2} e^{\varphi} \cos \theta \\
& \theta_{, 11}-\theta_{, 22}=2 q^{2} e^{\varphi} \sin \theta
\end{aligned}
$$

where the function $\varphi\left(u^{1}, u^{2}\right)$ determines the conformally-flat metric on the minimal surface $g_{11}=-g_{22}=e^{-\varphi}, g_{12}=0$ and $\theta\left(u^{1}, u^{2}\right)$ determines the second quadratic forms $B_{3 \mid i j}$ and $B_{4 \mid i j}(i, j=1,2)$ and the torsion vector $v_{34 \mid i}(i=1,2)$ by formulae (3.12), (3.13) and (3.15).

A special choice of normals $\eta_{3}^{\mu}$ and $\eta_{4}^{\mu}$ to the minimal surface allows one to derive directly from the general system (3.3)-(3.5) the system of two equations for two functions in contrast with papers $[8,9,11]$ in which the normals $\eta_{3}^{\mu}$ and $\eta_{4}^{\mu}$ are not fixed. In these papers it was necessary to introduce auxiliary functions $\alpha_{ \pm}$, which entered in the final equations only in the form of differences $\theta=\alpha_{+}-\alpha_{-}$.

The general solution of system (3.16) can be derived using our formulae (2.12) and (2.13)

$$
\begin{aligned}
e^{\varphi} & =\Lambda_{2}, \\
\frac{\theta}{2}=\arctan \frac{B_{4 \mid 12}}{B_{3 \mid 11}} & =\arctan \left(\frac{x_{; 12}^{2}}{x_{; 11}^{2}}\right)^{1 / 2}
\end{aligned}
$$

To express $\theta$ through arbitrary functions $f_{i}\left(u^{+}\right)$and $g_{i}\left(u^{-}\right)$explicitly, it is more convenient to use the first of equations (3.16) substituting into it (3.17) rather than formulae (3.18), (2.12) and (2.7). As a result we obtain

$$
\theta\left(u^{1}, u^{2}\right)=\arccos \Delta_{2} \text {, }
$$


where

$$
\begin{gathered}
\Delta_{n}=2 \frac{\sum_{i=1}^{n}\left(f_{i}+g_{i}\right) f_{i}^{\prime} \sum_{j=1}^{n}\left(f_{j}+g_{j}\right) g_{j}^{\prime}}{\sum_{i=1}^{n}\left(f_{i}+g_{i}\right)^{2}\left\{\sum_{k=1}^{n} f_{k}^{\prime 2} \sum_{j=1}^{n} g_{j}^{\prime 2}\right\}^{1 / 2}}-\frac{\sum_{i=1}^{n} f_{i}^{\prime} g_{i}^{\prime}}{\left\{\sum_{k=1}^{n} f_{k}^{\prime 2} \sum_{j=1}^{n} g_{j}^{\prime 2}\right\}^{1 / 2}}, \\
f_{i} \equiv f_{i}\left(u^{+}\right), g_{i} \equiv g_{i}\left(u^{-}\right) .
\end{gathered}
$$

In paper [9] the general solution of system (3.16) has been written down by reducing these equations to one Liouville equation for the complex function $\varphi+i \theta$.

The new system of three nonlinear equations, which has not been mentioned in the literature before, is derived by treating the two-dimensional minimal surfaces in the five-dimensional pseudo-Euclidean space within the given method. Choosing the normals $\eta_{3}$ and $\eta_{4}$ according to the above prescription, we get formulae (3.12)-(3.15). There appear two torsion vectors $v_{35 \mid i}$ and $v_{45 \mid i}, i=1,2$ in addition to the variables in the four-dimensional case. The Peterson-Codazzi equation (3.4) with $\alpha=5$ yields

$$
v_{35 \mid 2} \cos \frac{\theta}{2}=v_{45 \mid 1} \sin \frac{\theta}{2}, \quad v_{45 \mid 2} \sin \frac{\theta}{2}=v_{35 \mid 1} \cos \frac{\theta}{2} .
$$

To satisfy these equalities, we assume

$$
\begin{aligned}
& v_{35 \mid 2}=h\left(u^{1}, u^{2}\right) \sin \frac{\theta}{2}, \quad v_{45 \mid 1}=h\left(u^{1}, u^{2}\right) \cos \frac{\theta}{2}, \\
& v_{45 \mid 2}=p\left(u^{1}, u^{2}\right) \cos \frac{\theta}{2}, \quad v_{35 \mid 1}=p\left(u^{1}, u^{2}\right) \sin \frac{\theta}{2} .
\end{aligned}
$$

The Ricci equations (3.5) are written now as

$$
\begin{aligned}
& \theta_{, 11}-\theta_{, 22}-\left(h^{2}-p^{2}\right) \sin \theta=2 q^{2} e^{\varphi} \sin \theta \\
& \sin \frac{\theta}{2}\left(h_{, 1}-p_{, 2}\right)+\cos \frac{\theta}{2}\left(h \theta_{, 1}-p \theta_{, 2}\right)=0 \\
& \cos \frac{\theta}{2}\left(p_{, 1}-h_{, 2}\right)+\sin \frac{\theta}{2}\left(h \theta_{, 2}-p \theta_{, 1}\right)=0 .
\end{aligned}
$$

Substitution of

$$
h=\chi_{, 2}\left(\sin \frac{\theta}{2}\right)^{-2}, \quad p=\chi_{, 1}\left(\sin \frac{\theta}{2}\right)^{-2}
$$

turns equation (3.24) into an identity, and (3.25) gives

$$
\left(\cot ^{2} \frac{\theta}{2} \cdot \chi_{, 1}\right)_{, 1}=\left(\cot ^{2} \frac{\theta}{2} \cdot \chi_{, 2}\right)_{, 2} .
$$

Finally, in the case of the five-dimensional enveloping space system (3.3)-(3.5) 
is reduced to the following three nonlinear equations

$$
\begin{gathered}
\varphi_{, 11}-\varphi_{, 22}=2 q^{2} e^{\varphi} \cos \theta, \theta_{, 11}-\theta_{, 22}+2 \frac{\cos \frac{\theta}{2}}{\sin ^{3} \frac{\theta}{2}}\left(\chi_{, 1}^{2}-\chi_{, 2}^{2}\right)=2 q^{2} e^{\varphi} \sin \theta \\
\left(\cot ^{2} \frac{\theta}{2} \cdot \chi_{, 1}\right)_{.1}=\left(\cot ^{2} \frac{\theta}{2} \cdot \chi_{2}\right)_{, 2} .
\end{gathered}
$$

It should be emphasized that the last of equations (3.28) coincides exactly with the second equation in the nonlinear Lund-Regge system $[7,20]$.

Let us write down the general solution of system (3.28)

$$
\begin{aligned}
e^{\varphi} & =\Lambda_{3}, \quad \theta=\arccos \Delta_{3}, \\
\frac{\chi_{, 1}^{2}}{\sin ^{2} \frac{\theta}{2}} & =q^{2} \cos ^{2} \frac{\theta}{2} \cdot e^{\varphi}-\frac{\theta_{, 2}^{2}}{4}-\left[\frac{\partial}{\partial u^{1}}\left(\frac{x_{; 11}^{\mu}}{q \cos \frac{\theta}{2}}\right)\right]^{2}, \\
\frac{\chi_{, 2}^{2}}{\sin ^{2} \frac{\theta}{2}} & =-q^{2} \cos ^{2} \frac{\theta}{2} \cdot e^{\varphi}-\frac{\theta_{, 1}^{2}}{4}-\left[\frac{\partial}{\partial u^{2}}\left(\frac{x_{; 11}^{\mu}}{q \cos \frac{\theta}{2}}\right)\right]^{2} .
\end{aligned}
$$

The quantities $\Lambda_{3}$ and $\Delta_{3}$ are determined by (2.13) and (3.20), respectively, and the covariant derivative $x_{; 11}^{\mu}$ should be constructed by using expansions $(2.7)$ and (2.12).

It is obvious that the proposed method can be applied also for higher dimensions of the enveloping space. At the same time equations (3.3)-(3.5) will be reduced to the system of $(n-2)$ nonlinear equations for $n-2$ unknown functions. The general solutions of these systems will be constructed by formulae (2.13), (3.20) and expansions (2.7) and (2.12).

\section{Gauge $t=\tau$}

In this gauge the world surface of the string moving in the $n$-dimensional pseudo-Euclidean space is described by the $(n-1)$-dimensional Euclidean vector $\mathbf{x}=\left\{x^{1}, s^{2}, \ldots, x^{n-1}\right\}$ which depends on two parameters $\tau=t=x^{0}=u^{1}$ and $\sigma=u^{2}$. The equations of motion (2.4) and supplementary conditions (2.2) are

$$
\begin{gathered}
\mathbf{x}_{, 11}-\mathbf{x}_{, 22}=0 \\
\mathbf{x}_{, 1}^{2}+\mathbf{x}_{, 2}^{2}=1, \quad \mathbf{x}_{, 1} \mathbf{x}_{, 2}=0 .
\end{gathered}
$$

Conditions (4.2) cause the following form of the metric tensor on the world surface of the string

$$
g_{11}=\mathbf{x}_{, 1}^{2}=\sin ^{2} \theta, \quad g_{22}=\mathbf{x}_{, 2}^{2}=\cos ^{2} \theta, \quad g_{12}=\mathbf{x}_{, 1} \mathbf{x}_{, 2}=0 .
$$


In what follows we shall need the Christoffel symbols corresponding to this metric

$$
\begin{array}{ll}
\Gamma_{11}^{1}=\Gamma_{22}^{1}=\cot \theta \cdot \theta_{, 1}, & \Gamma_{12}^{1}=\Gamma_{21}^{1}=\cot \theta \cdot \theta_{, 2}, \\
\Gamma_{11}^{2}=\Gamma_{22}^{2}=-\tan \theta \cdot \theta_{, 2}, & \Gamma_{12}^{2}=\Gamma_{21}^{2}=-\tan \theta \cdot \theta_{, 1} .
\end{array}
$$

The world surface of the string in the gauge $t=\tau$ is not minimal for the $(n-1)$-dimensional Euclidean space with the coordinates $x^{1}, \ldots, x^{n-1}$. Nevertheless, from the derivative formulae (3.1) taking into account the equations of motion (4.1) and formulae (4.4), it follows that

$$
B_{\alpha \mid 11}=B_{\alpha \mid 22}, \quad \alpha=3,4, \ldots, n-1 .
$$

The only essential component of the Riemann curvature tensor in metric (4.3) has the form [8]

$$
R_{1212}=\frac{1}{2} \sin 2 \theta\left(\theta_{, 11}-\theta_{, 22}\right)
$$

Using (4.7) and (4.6) the Gauss equation (3.3) can be written as

$$
\frac{1}{2} \sin 2 \theta \cdot\left(\theta_{, 11}-\theta_{, 22}\right)=\sum_{\alpha=3}^{n-1}\left(B_{\alpha \mid 11}^{2}-B_{\alpha \mid 12}^{2}\right) .
$$

The construction of general solutions of equations (3.3)-(3.5) will be based, as in Sect. 3, on the solutions of equations of motion (4.1) satisfying conditions (4.2) in a special basis.

First consider the simplest case of the three-dimensional space-time with the relativistic string moving in it. In the gauge $t=\tau$ the string coordinates $x^{1}(t, \sigma)$ and $x^{2}(t, \sigma)$ determine the plane which is the projection of the world surface of the string in the space $\left\{t, x^{1}, x^{2}\right\}$ onto the coordinate plane $O x^{1} x^{2}$.

In this case $B_{\alpha \mid i j}=0$ and $v_{\alpha \beta \mid i}=0$, and the only nontrivial equation in system (3.3)-(3.5) is the Gauss equation which is reduced, according to (4.6), to the D'Alambert equation [8]

$$
\theta_{, 11}-\theta_{, 22}=0 \text {. }
$$

The solution of Eqs. (4.1) and (4.2) for the two-dimensional vector $\mathbf{x}(t, \sigma)$ is as follows:

$$
\begin{aligned}
\mathbf{x}(t, \sigma) & =\psi_{+}\left(u^{+}\right)-\psi_{-}\left(u^{-}\right), \quad\left(\psi^{\prime}\right)^{2}=1, \quad u^{ \pm}=t \pm \sigma, \\
\psi_{ \pm}^{\prime}\left(u^{ \pm}\right) & =\frac{1}{\sqrt{2}}\left\{\cos \varphi_{ \pm}\left(u^{ \pm}\right), \quad \pm \sin \varphi_{ \pm}\left(u^{ \pm}\right)\right\} .
\end{aligned}
$$

According to (4.3) the function $\theta(t, \sigma)$ is determined by the formula

$$
\theta(t, \sigma)=\arctan \left(\frac{\mathbf{x}_{, 1}^{2}}{\mathbf{x}_{, 2}^{2}}\right)^{1 / 2}
$$

Substituting into the latter formulae (4.9) and (4.10), we obtain the general solution of Eq. (4.8)

$$
\theta(t, \sigma)=\arctan \left[\frac{1-\cos \left(\varphi_{+}+\varphi_{-}\right)}{1+\cos \left(\varphi_{+}+\varphi_{-}\right)}\right]^{1 / 2}=\left[\varphi_{+}\left(u^{+}\right)+\varphi_{-}\left(u^{-}\right)\right] / 2 .
$$


This trivial case demonstrates clearly the idea of deriving general solutions of equations (3.3)-(3.5) in the gauge $t=\tau$.

Now we pass to four-dimensional space-time. In this case the three-component vector $\mathbf{x}(t, \sigma)$ describes the projection of the two-dimensional minimal surface from four-dimensional Minkowski space onto the ordinary three-dimensional Euclidean space. The system of equations (3.3)-(3.5), taking into account (4.4)-(4.7), is reduced, as it can easily be shown, to the following two nonlinear equations:

$$
\theta_{, 11}-\theta_{, 22}+\frac{\cos \theta}{\sin ^{3} \theta}\left(\chi_{.1}^{2}-\chi_{, 2}^{2}\right)=0, \quad\left(\cot ^{2} \theta \cdot \chi_{, 1}\right)_{, 1}=\left(\cot ^{2} \theta \cdot \chi_{, 2}\right)_{, 2},
$$

where the function $\chi$ determines the coefficients of the second quadratic form

$$
B_{11}=B_{22}=\cot \theta \cdot \chi_{, 2}, \quad B_{12}=\cot \theta \cdot \chi_{, 1} .
$$

The system (4.13) differs from the known Lund-Regge equations [20] by the absence of the term $\sin \theta \cdot \cos \theta$ in the first equation.

We represent again the vector $\mathbf{x}(t, \sigma)$ using formulae (4.9), and for $\psi_{ \pm}^{\prime}\left(u^{ \pm}\right)$we take the spherical basis in three-dimensional Euclidean space

$$
\begin{gathered}
\psi_{ \pm}^{\prime}\left(u^{ \pm}\right)=\frac{1}{\sqrt{2}}\left\{\sin \omega_{ \pm} \cdot \cos \varphi_{ \pm}, \pm \sin \omega_{ \pm} \cdot \sin \varphi_{ \pm}, \pm \cos \omega_{ \pm}\right\}, \\
\omega_{ \pm} \equiv \omega_{ \pm}\left(u^{ \pm}\right), \quad \varphi_{ \pm} \equiv \varphi_{ \pm}\left(u^{ \pm}\right) .
\end{gathered}
$$

For $\theta(t, \sigma)$ according to (4.11), we obtain

$\theta(t, \sigma)=\arctan \left\{\frac{1-\left[\sin \omega_{+} \cdot \sin \omega_{-} \cdot \cos \left(\varphi_{+}+\varphi_{-}\right)-\cos \omega_{+} \cdot \cos \omega_{-}\right]}{1+\left[\sin \omega_{+} \cdot \sin \omega_{-} \cdot \cos \left(\varphi_{+}+\varphi_{-}\right)-\cos \omega_{+} \cdot \cos \omega_{-}\right]}\right\}^{1 / 2}$.

Using the law of cosines for the spherical triangle [21], one can easily verify that $2 \theta(t, \sigma)$ in $(4.16)$ is the angle in the spherical triangle opposite the side $\varphi_{+}+\varphi_{-}$ with the adjoining angles $\omega_{+}$and $\omega_{-}$.

Only the partial derivatives of the function $\mathbf{x}(t, \sigma)$ enter into system (4.13). According to the derivative formulae (3.1), they are determined as follows:

$$
\chi_{, 1}=\tan \theta \cdot\left(\mathbf{x}_{; 12}^{2}\right)^{1 / 2}, \quad \chi_{2}=\tan \theta \cdot\left(\mathbf{x}_{; 11}^{2}\right)^{1 / 2} .
$$

It is obvious that expansions (4.9) and (4.15) allow one to express $\chi_{, i}, i=1,2$ through four arbitrary functions of one variable $\varphi_{+}\left(u^{ \pm}\right)$and $\omega_{ \pm}\left(u^{ \pm}\right)$. Therefore we shall not cite here these cumbersome formulae.

Generalizing representations $(4.10),(4.15)$ to the $n$-dimensional case, one can construct the general solutions of the system of nonlinear equations (3.3)-(3.5) in the gauge $t=\tau$ for an arbitrary dimension $n$ of the enveloping space.

\section{Conclusion}

It is certainly interesting to investigate the group basis of the proposed way of constructing general solutions for non-linear equations in the spirit of paper [22]. The same procedure, minimal embedding, is used in our approach and in 
paper [22]. In our case this is the minimal embedding of the two-dimensional Riemannian manifolds into the plane space, whereas in paper [22] it is the minimal embedding of one Lie group into another. Just the minimal embedding led to the linear equations (2.4) for the coordinates of the embedding submanifold. As a result this allows the construction of general solutions for the system of nonlinear equations (3.3)-(3.5).

\section{References}

1. Jackiw, R.: Rev. Mod. Phys. 49, 681 (1977)

2. Nambu, Y.: Phys. Rev. D10, 4262-4268 (1974); Phys. Rep. 23C, 250-253 (1976)

3. Faddeev, L. D., Korepin, V. E.: Phys. Rep. 42C, 1-87 (1978)

4. Barbashov, B. M., Nesterenko, V. V.: Phys. Elem. Part. Atom. Nucl. (in Russian) 9, 709-758 (1978)

5. Ablowitz, M. J., Kaup, D. J., Newell, A. C., Segur, H.: Phys. Rev. Lett. 30, 1262-1264; Stud. Appl. Math. 53, 249 (1974)

6. Sasaki, R.: Nucl. Phys. B154, 343-357 (1979)

7. Lund, F.: Phys. Rev. D15, 1540 (1977)

8. Barbashov, B. M., Nesterenko, V. V.: Fortschr. Phys. 28, 427-464 (1980)

9. Barbashov, B. M., Nesterenko, V. V., Chervyakov, A. M.: J. Phys. A13, 301-312 (1980)

10. Nesterenko, V. V.: Lett. Math. Phys. 4, 451-456 (1980)

11. Barbashov, B. M., Nesterenko, V. V.: Commun. Math. Phys. 78, 499-506 (1981)

12. Wilson, K. G.: Phys. Rev. D10, 2445-2459 (1974)

13. Favard, J.: Cours de geometrie differentialle locale. Paris: Gauthier-Villars 1957

14. Barbashov, B. M., Koshkarov, A. L.: Teor. Mat. Fiz. 39, 27-34 (1979)

15. Eisenhart, L. P.: Riemannian Geometry. Princeton: Princeton University Press 1964

16. Osserman, R.: Bull. Am. Math. Soc. 75, 1092-1120 (1969)

17. Omnes, R.: Nucl. Phys. B149, 269-284 (1979)

18. Bianchi, L.: Lezioni di Geometria Differenziale, $4^{\text {th }}$ ed. Bologna 1922-1923

19. Forsyth, A. R.: Theory of Differential Equations, v. 5-6. New York: Dover Publications 1959

20. Lund, F.: Ann. Phys. 115, 251-268 (1978)

21. Handbook of Mathematical Functions. Edited by Abramowitz, M. and Stegun, I. A. National Bureau of Standards Applied Mathematics Series 55, 1964

22. Leznov, A. N., Saveliev, M. V.: Phys. Elem. Part. Atom. Nucl. (in Russian) 11, 40-91 (1980); 12, $125-$ $161(1981)$

Communicated by H. Araki

Received June 22, 1981; in revised form October 14, 1981 
